\title{
AN OSCILLATION THEOREM FOR SECOND ORDER SUBLINEAR DIFFERENTIAL EQUATIONS
}

\author{
JAMES S. W. WONG
}

(Communicated by Kenneth R. Meyer)

\begin{abstract}
An oscillation criterion is given for the second order sublinear differential equation $x^{\prime \prime}+a(t)|x|^{\gamma} \operatorname{sgn} x=0,0<\gamma<1$, where the coefficient $a(t)$ is not assumed to be nonnegative for all large values of $t$. The result extends a condition recently discovered by Butler, Erbe, and Mingarelli for the linear equation.
\end{abstract}

Consider the second order sublinear differential equation

$$
x^{\prime \prime}+a(t)|x|^{\gamma} \operatorname{sgn} x=0, \quad 0<\gamma<1,
$$

where $a(t) \in C[0, \infty)$. We restrict our attention to solutions of (1) which exist on some ray $\left[t_{0}, \infty\right)$, where $t_{0} \geqq 0$ may depend on the particular solution. Such a solution is said to be oscillatory if it has arbitrarily large zeros. Equation (1) is called oscillatory if all such solutions are oscillatory. For a general discussion on sublinear oscillation problems, we refer the reader to [11]. We are here concerned with sufficient conditions on $a(t)$ for the oscillation of (1) when $a(t)$ is allowed to assume negative values for arbitrarily large values of $t$. In the linear case, the well-known Wintner's oscillation theorem states that if $a(t)$ satisfies

$$
\lim _{T \rightarrow \infty} \frac{1}{T} \int_{0}^{T} \int_{0}^{t} a(s) d s d t=+\infty,
$$

then equation (1) is oscillatory for $\gamma=1$, see [10]. Butler [1] proved that Wintner's theorem remains valid for equation (1) where $\gamma>1$. In the sublinear case, i.e., $0<\gamma<1$, condition (2) can be relaxed to

$$
\limsup _{T \rightarrow \infty} \frac{1}{T} \int_{0}^{T} \int_{0}^{t} a(s) d s d t=+\infty,
$$

an earlier result due to Kamenev [6]. We note that Condition (3) alone is not sufficient for oscillation for $\gamma \geq 1$; in the linear case, see Willett [9].

Received by the editors January 27, 1989.

1980 Mathematics Subject Classification (1985 Revision). Primary 34C10, 34C15.

Key words and phrases. Second order, sublinear, ordinary differential equations, oscillation, weighted averages. 
Wintner's condition (2) was later improved by Hartman [3], see also [4], who proved that the following conditions, i.e.,

$$
\liminf _{T \rightarrow \infty} \frac{1}{T} \int_{0}^{T} A(t) d t>-\infty,
$$

where $A(t)=\int_{0}^{t} a(s) d s$ and that the limit in (2) does not exist, namely,

$$
\varliminf_{T \rightarrow \infty} \frac{1}{T} \int_{0}^{T} A(t) d t<\varlimsup_{T \rightarrow \infty} \frac{1}{T} \int_{0}^{T} A(t) d t,
$$

are sufficient for oscillation of the linear equation. In particular, conditions (3) and (4) form an oscillation criterion for equation (1) with $\gamma=1$. Butler [1] also proved that in the sublinear case, i.e., equation (1), condition (5) alone will suffice, (see also Kwong and Wong [8, Theorem 6], and Wong [14] for alternative proofs of this result). Recently in connection with the study of oscillation theory for linear systems, Butler, Erbe, and Mingarelli [2] showed that condition (4), together with

$$
\limsup _{T \rightarrow \infty} \frac{1}{T} \int_{0}^{T} A^{2}(t) d t=+\infty,
$$

are sufficient for oscillation of equation (1) in the linear case. Since (3) implies (6) by an application of Schwarz's inequality, their result extends part of Hartman's theorem, i.e., condition (3) plus (4), and is hence a new oscillation criterion thus far undiscovered. It is therefore natural to ask whether this new oscillation criterion remains valid for the sublinear equation (1). The purpose of this note is to answer this question in the affirmative by proving

Theorem. Suppose that conditions (4) and (6) hold. Then equation (1) is oscillatory for $0<\gamma<1$.

Proof. Assume to the contrary that there exists a nonoscillatory solution $x(t)$, which may be assumed to be positive on $\left[t_{0}, \infty\right)$. For $0<\gamma<1$, define $y(t)=x^{1-\gamma}(t)$. It is easy to verify from (1) that $y(t)$ satisfies the second order nonlinear differential equation

$$
y^{\prime \prime}+(1-\gamma) a+\gamma(1-\gamma)^{-1} y^{-1} y^{\prime 2}=0,
$$

on $\left[t_{0}, \infty\right)$. For convenience, denote the positive numbers $\alpha=1-\gamma, \beta=$ $\gamma(1-\gamma)^{-1}$. Integrating (7) once obtains

$$
y^{\prime}(t)-y^{\prime}\left(t_{0}\right)+\alpha \int_{t_{0}}^{t} a(s) d s+\beta \int_{t_{0}}^{t} y^{-1}(s) y^{\prime 2}(s) d s=0 .
$$

A further integration yields

$$
y(t)-y\left(t_{0}\right)+\beta \int_{t_{0}}^{t} \int_{t_{0}}^{s} y^{-1} y^{\prime 2}=C_{0}\left(t-t_{0}\right)-\alpha \int_{t_{0}}^{t} A(s) d s,
$$

where $C_{0}=y^{\prime}\left(t_{0}\right)+\alpha A\left(t_{0}\right)$. 
We distinguish two mutually exclusive cases, namely (i) $y^{-1} y^{\prime 2} \notin L^{1}\left(T_{0}, \infty\right)$ and (ii) $y^{-1} y^{\prime 2} \in L^{1}\left(t_{0}, \infty\right)$, and verify in each case that the existence of a positive solution leads to a contradiction. If $y^{-1} y^{\prime 2} \notin L^{1}\left(t_{0}, \infty\right)$, then the weighted average also diverges, i.e.,

$$
\lim _{t \rightarrow \infty} \frac{1}{t} \int_{t_{0}}^{t} \int_{t_{0}}^{s} y^{-1} y^{\prime 2}=+\infty
$$

Dividing (9) by $t$, noting that $y(t)$ is positive and using (10) and condition (4), we easily arrive at a desired contradiction.

We now turn to the case when $y^{-1} y^{\prime 2} \in L^{1}\left(t_{0}, \infty\right)$, and first show that

$$
\lim _{t \rightarrow \infty} \frac{y(t)}{t}=0 \text {. }
$$

Let $\varepsilon>0$, choose $t_{1} \geq t_{0}$ such that $\int_{t_{1}}^{\infty} y^{-1} y^{\prime 2}<\frac{\varepsilon}{4}$. By Schwarz's inequality,

$$
y(t)-y\left(t_{1}\right)=\int_{t_{1}}^{t} y^{\prime} \leq\left(\int_{t_{1}}^{t} y^{-1} y^{\prime 2}\right)^{1 / 2} y\left(\int_{t_{1}}^{t} y\right)^{1 / 2} y \leq \frac{\sqrt{\varepsilon}}{2}\left(\int_{t_{1}}^{t} y\right)^{1 / 2} y .
$$

Suppose that $y \in L^{1}\left(t_{1}, \infty\right)$. Then (12) implies $y(t)$ is bounded, hence (11) follows. Thus we assume that $y \notin L^{1}\left(t_{1}, \infty\right)$, so one can choose $t_{2} \geq t_{1}$ so that $y^{2}\left(t_{1}\right) \leq \frac{\varepsilon}{4} \int_{t_{1}}^{t} y$, for $t \geq t_{2}$. Using this fact in (12),

$$
y(t) \leq y\left(t_{1}\right)+\frac{\sqrt{\varepsilon}}{2}\left(\int_{t_{1}}^{t} y\right)^{1 / 2} y \leq\left(\frac{\sqrt{\varepsilon}}{2}+\frac{\sqrt{\varepsilon}}{2}\right)\left(\int_{t_{1}}^{t} y\right)^{1 / 2} y .
$$

Dividing (13) through by $\left(\int_{t_{1}}^{t} y\right)^{1 / 2} y$ and integrating from $t_{2}$ to $t$ obtains

$$
\left(\int_{t_{1}}^{t} y\right)^{1 / 2} y-\left(\int_{t_{1}}^{t_{2}} y\right)^{1 / 2} y \leq \frac{\sqrt{\varepsilon}}{2}\left(t-t_{2}\right) \leq \frac{\sqrt{\varepsilon}}{2} t .
$$

Now choose $t_{3} \geq t_{2}$ such that $\int_{t_{1}}^{t_{2}} y \leq \frac{\varepsilon}{4} t_{3}^{2}$, hence, for $t \geq t_{3}$, we have from (14),

$$
\left(\int_{t_{1}}^{t} y\right)^{1 / 2} y \leq \frac{\sqrt{\varepsilon}}{2} t+\frac{\sqrt{\varepsilon}}{2} t_{3} \leq\left(\frac{\sqrt{\varepsilon}}{2}+\frac{\sqrt{\varepsilon}}{2}\right) t .
$$

Combining (13) and (15), we conclude that $y(t) \leq \varepsilon t$ for $t \geq t_{3}$, proving (11).

Returning to (8), one can express $A(t)$ in the form

$$
\alpha A(t)=y^{\prime}\left(t_{0}\right)-y^{\prime}(t)+\alpha A\left(t_{0}\right)-\beta \int_{t_{0}}^{t} y^{-1} y^{\prime 2} .
$$

Recalling that $C_{0}=y^{\prime}\left(t_{0}\right)+\alpha A\left(t_{0}\right)$, one can use (16) to estimate $A^{2}(t)$ by

$$
\alpha^{2} A^{2}(t) \leq 3 C_{0}^{2}+3 y^{\prime 2}(t)+3 \beta^{2} \int_{t_{0}}^{t} y^{-1} y^{\prime 2} \leq 3 C_{1}^{2}+3 y^{\prime 2}(t),
$$


where $C_{1}^{2}=C_{0}^{2}+\beta^{2} \int_{t_{0}}^{\infty} y^{-1} y^{\prime 2}$. Integrating (17) from $t_{0}$ to $T$ and dividing through by $T$, we obtain

$$
\frac{\alpha^{2}}{T} \int_{t_{0}}^{T} A^{2}(t) d t \leq 3 C_{1}^{2}\left(1-\frac{t_{0}}{T}\right)+\frac{3}{T} \int_{t_{0}}^{T} y^{\prime 2} .
$$

Finally, we note

$$
\frac{1}{T} \int_{t_{0}}^{T} y^{\prime 2} y^{-1} y \leq \frac{1}{T} \max _{t_{0} \leq t \leq T}|y(t)| \int_{t_{0}}^{T} y^{-1} y^{\prime 2} .
$$

By (11) we can choose $T_{0} \geq t_{0}$ such that $|y(t)| \leq t$ for $t \geq T_{0}$. Using this,

$$
\max _{t_{0} \leq t \leq T}|y(t)| \leq \max _{t_{0} \leq t \leq T_{0}}|y(t)|+T \leq M_{0}+T \text {, }
$$

where $M_{0}$ is a constant of $T$. Substituting (19) and (20) in (18), we find the right hand side is bounded as $T$ tends to infinity, which is incompatible with condition (6).

Remark 1. It is easy to give an example of $a(t)$ which satisfies conditions (4) and (6) but fails to satisfy conditions (3) and (4). Take $A(t)=t^{\lambda} \sin t, 1 / 2<$ $\lambda<1$. We note in passing that condition (5) alone suffices for the oscillation of equation (1) in the sublinear case, so does condition (3). Nevertheless, the example

$$
a(t)=t^{\lambda} \cos t+\lambda t^{\lambda-1} \sin t, \quad \frac{1}{2}<\lambda<1
$$

does not satisfy either (3) or (5), but our theorem applies.

Remark 2. We note that the weighted average used here is the simple arithmetic mean used by Wintner [10] in condition (2). Hartman [5] showed that some of his results, which include that of Wintner, remain valid for a much wider class of "general means". It would be interesting to investigate to what extent oscillation criteria with more general weighted means for the linear equation can be extended to the sublinear case. Some of the oscillation theorems using weighted means by Willett [9] have been successfully extended by Butler [1].

Remark 3. Condition (3) has been extended by Kamenev [7] to the weaker condition for the linear equation, i.e., for some $\mu>1$,

$$
\limsup _{T \rightarrow \infty} \frac{1}{T^{\mu}} \int_{0}^{T}(T-t)^{\mu} a(t) d t=+\infty .
$$

It is known that (21) is also sufficient for the sublinear equation, see [13].

\section{REFERENCES}

1. G. J. Butler, Integral averages and the oscillation of second order ordinary differential equations, SIAM J. Math. Anal. 11 (1980), 190-200.

2. G. J. Butler, L. H. Erbe, and A. B. Mingarelli, Riccati techniques and variational principles in oscillation theory for linear systems, Trans. Amer. Math. Soc. 303 (1987), 263-282.

3. P. Hartman, On nonoscillatory linear differential equations of second order, Amer. J. Math. 74 (1952), 389-400. 
4. __ Ordinary differential equations, 2nd. ed., Wiley, New York, 1973.

5. - On nonoscillatory linear differential equations of second order, Proc. Amer. Math. Soc. 64 (1977), 251-259.

6. I. V. Kamenev, Certain specifically nonlinear oscillation theorems, Mat. Zametki 10 (1971), 129-134. (Russian)

7. _ Integral criterion for oscillations of linear differential equations of second order, Mat. Zametki 23 (1978), 249-251. (Russian)

8. M. K. Kwong and J. S. W. Wong, Linearization of second order nonlinear oscillation theorems, Trans. Amer. Math. Soc. 279 (1983), 705-722.

9. D. Willett, Classification of second order linear differential equations with respect to oscillation, Adv. in Math. 3 (1969), 594-623.

10. A. Wintner, A criterion of oscillatory stability, Quart. Appl. Math. 7 (1949), 114-117.

11. J. S. W. Wong, Oscillation theorems for second order nonlinear differential equations, Bull. Inst. Math. Acad. Sinica 3 (1975), 283-309.

12. An oscillation criterion for second-order nonlinear differential equations, Proc. Amer. Math. Soc. 98 (1986), 109-112.

13. __ An oscillation criterion for second-order sublinear differential equations, Conference Proceedings, Canadian Mathematical Society, vol. 8, 1987, pp. 299-302.

14. __ Oscillation theorems for second-order nonlinear ordinary differential equations, Proc. Amer. Math. Soc. 106 (1989), 1069-1077.

Chinney Investments Ltd, 814 Swire House, Hong Kong 